\title{
Survival and Predictors of Mortality among Severe Acute Malnourished under-five children admitted at Felege-Hiwot Comprehensive Specialized Hospital, Northwest, Ethiopia: A retrospective cohort study
}

Amare Kassaw ( $\sim$ amarekassaw2009@gmail.com )

Debre Tabor University

Desalegne Amare

Bahir Dar University

Minyichil Birhanu

Bahir Dar University

Aragaw Tesfaw

Debre Tabor University

Shegaw Zeleke

Debre Tabor University

Getachew Arage

Debre Tabor University

Demewoz Kefale

Debre Tabor University

Research Article

Keywords: Mortality, Severe Acute Malnutrition, Survival, Under-five children

Posted Date: December 29th, 2020

DOl: https://doi.org/10.21203/rs.3.rs-124227/v1

License: (c) (i) This work is licensed under a Creative Commons Attribution 4.0 International License.

Read Full License

Version of Record: A version of this preprint was published at BMC Pediatrics on April 16th, 2021. See the published version at https://doi.org/10.1186/s12887-021-02651-x. 


\section{Abstract}

Background Malnutrition is still a global public health problem contributing for under-five morbidity and mortality. Specifically, childhood mortality attributable to malnutrition is high at Felege-Hiwot Comprehensive Specialized Hospital. However, there is no recent evidence that shows the time to death and public health importance of oxygen saturation and chest in drawing in the hospital. Therefore, estimated time to death and its predictors can provide an input for program planners and decisionmakers in the study area.

Methods An institutional-based retrospective cohort study was conducted among 488 severe acute malnourished under-five children admitted from the 1st of January 2016 to the 30th of December 2019. The study participants were selected by using simple random sampling technique. Data were entered in to Epi-Data version 3.1 and exported to STATA version 15 statistical software for further analysis. The Kaplan Meir was used to estimate cumulative survival probability and a log-rank test was used to compare the survival time between different categories of explanatory variables. The Cox-proportional hazard regression model was fitted to identify predictors of mortality. P-value $<0.05$ was used to declare statistical significance.

Results Out of the total 488 randomly selected charts of children with severe acute malnutrition, 476 records were included in the final analysis. A total of $54(11.34 \%)$ children died with an incidence rate of 9.1death /1000 person- days. Failed appetite test (AHR: 2.4; 95\%Cl: 1.26, 4.67), altered consciousness level at admission (AHR: 2.4; 95\% Cl: 1.08, 4.67), oxygen saturation below 90\% (AHR: 3.3; 95\%Cl: 1.40, 7.87), edema (AHR 2.9; 95\% Cl: 1.45, 5.66) and HIV infection (AHR: $2.8 ; 95 \% \mathrm{Cl}: 1.24,6.36)$ were predictors of mortality for children diagnosed with severe acute malnutrition.

Conclusion The overall survival status of severe acute malnourished children was low as compared to national sphere standards and previous reports in the literature. The major predictors of mortality were oxygen saturation below $90 \%$, altered consciousness, HIV infection, edema and failed appetite test. Therefore, early screening of complications, close follow up and regular monitoring of sever acute malnourished children might improve child survival rate.

\section{Background}

Malnutrition refers to all deviations from adequate nutrition and jeopardizes children's survival, health, growth and development. It exists in two forms; over nutrition and undernutrition of macronutrients and micronutrients $(1,2)$. In the developing world undernutrition or protein-energy malnutrition is the common problem in acute or chronic form that profoundly affects children's survival(3). Undernutrition comprises of wasting, stunting and underweight (4). Severe acute malnutrition (wasting) is defined as very low weight for length/height (WFL/WFH) below - 3 z scores of the median World Health Organization (WHO) growth standards or less than $70 \%$ of the median National Center for Health Statistics standard or the presence of nutritional edema(5). 
Severe acute malnutrition remains the major cause of child morbidity and mortality worldwide $(1,2)$. In 2017, 51 million children under-five years were wasted and 16 million were severely wasted globally (6). Annually about 7.6 million deaths among children under-five years of age(7), from these $35 \%$ deaths were nutritional related and $4.4 \%$ of deaths were specifically attributable to severe wasting(8). The status of the world's children 2019 report showed that nearly half of all death in children under-five years attributed to undernutrition; it puts children at greater risk of dying from common infections, increases the frequency, severity of such infections and delays recovery time. Children with severe acute malnutrition are nearly 12 times more likely to die than healthy children(3).

Globally, it is estimated that around 1 to 2 million children die every year due to severe acute malnutrition. Most of them live in South Asia and Sub-Saharan Africa $(9,10)$. The mortality rate due to SAM in SubSaharan Africa ranges from 9.8-46\% (11-14). Evidences indicated that the likely cause of high mortality rate could be HIV infection $(11,15)$, lack of appetite, chest in drawing(14), co-morbidities and altered consciousness at admission $(16,17)$.

Despite Ethiopia is planning and implementing National Newborn and Child Survival Strategy to reduce under-five mortality from $64 / 1,000$ to $29 / 1,000$ by 2019/2020(18), 2019 Mini Ethiopia Demographic and Health survey report indicated that under-five mortality is $55 / 1,000(19)$. Malnutrition is the major contributor to mortality in Ethiopia being an underlying cause for nearly $45 \%$ of under-five deaths(18).

Even though Ethiopia uses a standard protocol for the management of severe acute malnutrition, it is the third leading cause of mortality in under-five years old children and more than one-fourth of deaths are occurring during hospital admission(20,21).

Several studies conducted in Ethiopia showed that HIV infection, shock, anemia and TB were significant predictors of mortality and low survival $(16,20,22,23)$. However, these studies did not consider factors like low oxygen saturation, chest in drawing and fast breathing which have significant effect on child mortality admitted with $\operatorname{SAM}(14,24)$. Determining the magnitude and predictor of morality is a vital issue. Therefore, this study can contribute to the provision of data that is essential in planning, prioritize budgeting and staff training that improves the management system of public health settings and reduce child mortality.

\section{Methods}

\section{Study area and period}

This study was conducted at Felege Hiwot Comprehensive Specialized Hospital. The hospital is found in Bahir Dar city which is the capital city of Amhara regional state and located approximately $565 \mathrm{~km}$ Northwest of Addis Ababa, the capital city of Ethiopia. The governmental health institutions in the city include one Specialized Comprehensive Hospital, teaching hospital, primary hospital and 10 health centers. The pediatric ward has 47 beds and an emergency unit with 10 resuscitation beds. It has a total 
of 45 health professionals (4 pediatricians, 8 general practitioners and 33 nurses). The study was conducted from February 27 to March 15, 2020.

\section{Study design and participant characteristics}

An institution-based retrospective follow up study was conducted. All under-five children diagnosed with severe acute malnutrition who were admitted during January 2016- December 2019 at FHCSH included in the study. However severe acute malnourished under-five children with incomplete records were excluded from the study.

\section{Sample size determination and sampling procedure}

The sample size was calculated by using Epi- info Version 7 statistical software with the assumptions of $95 \% \mathrm{Cl}, 5 \%$ margin of error, $80 \%$ power, exposed to unexposed ratio $1: 1,10 \%$ non-response rate and an important variable of the study (passed appetite test) (P1):4\% and adjusted hazard ratio (AHR:2.75) (25). The total under-five years aged SAM children admitted to TFU from January 2016-December 2019 at FHCSH were 1340. Unique chart numbers of SAM children were written on Microsoft excel. Then Simple random sampling technique was applied to select individual records using computer generation method and a random sample of 488 SAM children were selected.

\section{Data collection tools and procedure}

The data were collected by three trained professional nurses through document review with a structured checklist. The checklists were consisted socio-demographic, co-morbidities, feeding and treatment related factors which are adopted and modified from different related studies $(20,23,26)$, reviewing the medical charts and severe acute malnutrition management protocol guideline. The starting point for retrospective follow-up study was the time from first admission date and the endpoint was date of death and censored. The survival status of patients was obtained from the medical records. Survival time was calculated as the time between the dates of admission to the date of death, censored. All medical records of severe acute malnourished under-five children that fulfill the inclusion criteria who were admitted to TFU at FHCSH from the first of January, 2016 to December 30th, 2019 were retrospectively reviewed.

\section{Data Quality Assurance}

Data quality was assured by designing proper data extraction tools. The adopted data extraction tools were evaluated by experienced researchers. A pretest was done on $5 \%$ of the sample size with a structured checklist at FHCSH before starting the actual study period and necessary corrections were done. After the pretest, unrecorded variables were reduced from the data extraction tools and others were arranged as per medical records. Training was provided for data collectors and supervisor before data collection and there was close follow up of data collectors by supervisor and the principal investigator. The collected data were checked out for completeness, accuracy and clarity by the principal investigator and supervisor.

\section{Operational definition of terms}

\section{Censored}


Severe acute malnourished under-five children at TFU with predictors but recovered and discharged to home, discharged against medical advice or transfer out to other health institutions without knowing the outcome(20).

\section{Survival status}

Is the final outcome of severe acute malnourished children either death or censored.

\section{Event}

Death

\section{Co-morbidities}

Medical complications that will occur as a result of severe acute malnutrition (diarrhea, tuberculosis, pneumonia, CHF, anemia, shock, and hypoglycemia).

\section{Survival time}

Measures the follow-up of time from a defined starting point/from admission of under-five children diagnosed with SAM to TFU up to the occurrence of the event.

\section{Follow up time}

From the time of admission of under-five children diagnosed with SAM until either an event or censorship occurs.

\section{Length of stay}

The number of days the child stayed in the hospital from admission until death or censoring(4).

\section{Data processing and analysis}

Before analysis, the collected data were cleaned, coded and entered in to Epi-Data Version 3.1 and exported to STATA 15 statistical software. Multiple imputations were done for handling missing values. The continuous data, depending on the distribution, were described either in mean and standard deviation or median. Frequency distribution was used for categorical data.

Finally, the outcome of each participant was dichotomized into censored or death. Incidence density rate (IDR) was calculated for the entire study period. Kaplan Meir was used to estimate mean survival time and cumulative survival probability and log-rank test was used to compare the survival time between different categories of explanatory variables.

Before running Cox proportional hazard regression model multi-collinearity was checked using variance inflation factor (VIF) and with pair-wise correlation. Residuals also were checked using goodness-of-fit test by Cox Snell residuals, which was satisfied the model test. 
Proportional Hazard assumption was checked using the Schoenfeld residual statistical test.

Bi-variable Cox-proportional hazard regression model was fitted for each explanatory variable. Variables having $p$-value $<0.2$ in the bi-variable analysis was fitted to the multivariable Cox-proportional hazards regression model. Hazard ratio with $95 \%$ confidence interval and $p$-value $<0.05$ was used to measure the strength of association and to identify statistical significant predictors.

\section{Results}

\section{Socio-demographic characteristics}

Out of the total 488 randomly selected charts of SAM children, $476(97.5 \%)$ records were fulfilling enrollment criteria in the final analysis; and the remaining 12(2.5\%) were excluded (8 incomplete data and 4 charts were lost during data collection). Out of them more than half $245(51.5 \%)$ of the children enrolled in the study were males. Two-third of the participants $321(67.4 \%)$ came from rural area. The median age of children was 14 months with $(\mathrm{IQR}=19)$ and majority of $345(72.5 \%)$ were aged less than 24 months (Table 1). 
Table 1

Socio-demographic characteristics and nutritional status of SAM children admitted at FHCSH from 2016-2019, Northwest Ethiopia, 2020 $(n=476)$

\begin{tabular}{|c|c|c|}
\hline Variables & Categories & Frequency $\mathrm{n}(\%)$ \\
\hline \multirow[t]{2}{*}{ Age in month } & $<24$ & $345(72.5)$ \\
\hline & $\geq 24$ & $131(27.5)$ \\
\hline \multirow[t]{2}{*}{ Sex of the child } & Male & $245(51.5)$ \\
\hline & Female & $231(48.5)$ \\
\hline \multirow[t]{2}{*}{ Residence } & Urban & 155(32.6) \\
\hline & Rural & $321(67.4)$ \\
\hline \multirow[t]{3}{*}{ Type of SAM(N = 472) } & Marasmus & $278(58.9)$ \\
\hline & Kwashiorkor & $130(27.5)$ \\
\hline & marasmus-kwash & $64(13.6)$ \\
\hline \multirow[t]{2}{*}{$\mathrm{WHZ}(\mathrm{n}=470)$} & Z-score $\geq-3$ & 182(38.7) \\
\hline & Z-score $<-3$ & $288(61.3)$ \\
\hline \multirow[t]{2}{*}{$\mathrm{HAZ}(\mathrm{n}=471)$} & Z-score $<-3$ & $246(52.2)$ \\
\hline & Z-score $\geq-3$ & $225(47.8)$ \\
\hline \multirow[t]{2}{*}{ WAZ $(n=470)$} & Z-score $<-3$ & 150(31.9) \\
\hline & Z-score $\geq-3$ & $320(68.1)$ \\
\hline
\end{tabular}

\section{Routine medication and feeding-related characteristics}

Four hundred sixty-five (97.6\%) children had taken routine antibiotics, 337(70.8\%) and 206 (43.2\%) had received vitamin $A$ and Zink supplementation. About three fourth of the children had given nutritional therapy (F-75 and F-100) and 121(25.7\%) failed appetite test (Table 2). 
Table 2

Treatment and feeding patterns of SAM children admitted at FHCSH from 2016-2019, Northwest Ethiopia, 2020( $n=476)$

\begin{tabular}{|c|c|c|}
\hline Variables & Categories & Frequency $n(\%)$ \\
\hline \multirow[t]{2}{*}{ Routine antibiotics } & Yes & $465(97.7)$ \\
\hline & No & $11(2.3)$ \\
\hline \multirow[t]{2}{*}{ Intake of $F-100(n=474)$} & Yes & $357(75.3)$ \\
\hline & No & $117(24.7)$ \\
\hline \multirow[t]{2}{*}{ Intake of F-75(475) } & Yes & $353(74.3)$ \\
\hline & No & $122(25.7)$ \\
\hline \multirow[t]{2}{*}{ Folic acid given } & Yes & $363(76.3)$ \\
\hline & No & $113(23.7)$ \\
\hline \multirow[t]{2}{*}{ Vitamin A given } & Yes & $337(70.8)$ \\
\hline & No & 139(29.2) \\
\hline \multirow[t]{2}{*}{ Nasogastric feeding $(n=472)$} & Yes & $144(30.5)$ \\
\hline & No & $328(69.5)$ \\
\hline \multirow[t]{2}{*}{ Blood transfusion $(n=474)$} & Yes & $57(12.0)$ \\
\hline & No & $417(88.0)$ \\
\hline \multirow[t]{2}{*}{ Appetite test $(n=471)$} & Failed & $121(25.7)$ \\
\hline & Passed & $350(74.3)$ \\
\hline
\end{tabular}

\section{Clinical conditions and major co- morbidities}

Most of the children were in critical condition at the time of admission. The common co- morbidities identified during admission were anemia (43.8\%), diarrhea (40.9\%), pneumonia (40.7\%) pulmonary TB (13.0\%) and CHF (12.5\%) (Table 3) 
Table 3

Clinical conditions and major co-morbidities of SAM children admitted at FHCSH from 2016-2019, Northwest Ethiopia, 2020 $(n=476)$

\begin{tabular}{|c|c|c|}
\hline Variables & Categories & Frequency n (\%) \\
\hline \multirow[t]{2}{*}{ HIV/AIDS(n = 376) } & Reactive & $31(8.3)$ \\
\hline & Non-reactive & $345(91.7)$ \\
\hline \multirow[t]{2}{*}{ Pneumonia $(\mathrm{n}=472)$} & Yes & 192(40.7) \\
\hline & No & $280(59.3)$ \\
\hline \multirow[t]{2}{*}{ Pulmonary TB(n = 471) } & Yes & $61(13.0)$ \\
\hline & No & $410(87.0)$ \\
\hline \multirow[t]{2}{*}{$\mathrm{CHF}(\mathrm{n}=473)$} & Yes & $59(12.5)$ \\
\hline & No & $414(87.5)$ \\
\hline \multirow[t]{2}{*}{ UTI(n = 475) } & Yes & $35(7.4)$ \\
\hline & No & $437(92.6)$ \\
\hline \multirow[t]{2}{*}{ Hypoglycemia(n = 471) } & Yes & $45(9.6)$ \\
\hline & No & $426(90.4)$ \\
\hline \multirow[t]{2}{*}{$\operatorname{Shock}(n=471)$} & Yes & $16(3.4)$ \\
\hline & No & $455(96.6)$ \\
\hline \multirow[t]{2}{*}{ Chest in drawing $(\mathrm{n}=472)$} & Yes & $107(22.7)$ \\
\hline & No & $365(77.3)$ \\
\hline \multirow[t]{2}{*}{ Oxygen saturation $(n=472)$} & $<90 \%$ & $181(38.4)$ \\
\hline & $\geq 90 \%$ & $291(61.6)$ \\
\hline \multirow[t]{2}{*}{ Diarrhea $(n=472)$} & Yes & 192(40.7) \\
\hline & No & $280(59.3)$ \\
\hline \multirow[t]{2}{*}{ Anemia(hgb < 11 mg/dl) } & Yes & $206(43.8)$ \\
\hline & No & $264(56.2)$ \\
\hline \multirow[t]{2}{*}{ Fast breathing $(\mathrm{n}=472)$} & Yes & 144(30.51) \\
\hline & No & $328(69.5)$ \\
\hline \multirow[t]{2}{*}{ Admission Pulse rate } & Altered & $273(57.4)$ \\
\hline & Normal & $203(42.6)$ \\
\hline
\end{tabular}




\begin{tabular}{|lll|}
\hline Variables & Categories & Frequency $\mathbf{n}(\%)$ \\
\hline Admission respiratory rate & Altered & $214(45.0)$ \\
\cline { 2 - 3 } & Normal & $262(55.0)$ \\
\hline \multirow{2}{*}{ Status at admission $(\mathrm{n}=472)$} & Altered & $27(5.7)$ \\
\cline { 2 - 3 } & Conscious & $445(94.3)$ \\
\cline { 2 - 3 } &
\end{tabular}

\section{Survival status of severe acute malnourished children}

A total of 476 were followed for different periods: a minimum of 2 and a maximum of 46 days and overall median hospital stay were 11 days. Of 476 children whose recorded were reviewed, $54(11.3 \%)$ with (95\% Cl: $8.40,14.30)$ died and $422(88.7 \%)$ censored (among them 324(68.1\%) recovered $14(2.9 \%)$ were referred, $36(7.6 \%)$ defaulted and $48(10.1 \%)$ were against medical advice at the end of the study period. The study participants were followed for 5936 person -day with an incidence rate of 9.1 death per 1000 person- day observation (95\% Cl: $6.97,11.88)$.

This study also showed that the incidence of mortality in HIV infected children was higher than HIV uninfected with an incidence rate of $25.7 \% / 1000(95 \% \mathrm{Cl}$ : $15.00,44.00)$. The incidence of mortality in SAM children who were impaired consciousness during admission was also higher compared to conscious with an incidence rate of $44.4 \% / 1000$ (95\% Cl: $27.00,72.00)$.

\section{Overall survival function}

The survival probability of severe acute malnourished children was estimated using Kaplan-Meier estimate. The survival probability in the second day of admission was high almost (99.6\%) with a standard error of $0.003(95 \% \mathrm{Cl}: 98,99.9)$. At the 12 th day of hospital stay the survival probability of SAM children was also found to be $90.9 \%$ with a standard error of $0.0156(95 \% \mathrm{Cl}: 87.00,94.00)$, from 20 to 25 days of hospital stay the probability of surviving was $72.8 \%$ with a standard error of $0.0424(95 \% \mathrm{Cl}: 63.00$, $80.00)$ and at the end of 45 days the overall survival probability was $59.2 \%$ with standard error $0.099(95 \%$ Cl: 38.00, 76.00) (Fig. 1).

\section{Survival function and Comparison of Survivorship Functions}

In this study, SAM children who failed appetite test at admission had lower survival time compared to those who passed appetite test. At the 35 days of hospital stays the cumulative survival probability of SAM children who failed appetite test were $32.4 \%$ as compared to those passed appetite test $(84.1 \%)$ (Fig. 2).

The survival time of SAM children with oxygen saturation less than $90 \%$ was also lower than those with saturation greater than $90 \%$. The overall survival at the end of the follow up period was $44.2 \%$ for those 
who had oxygen saturation $<90 \%$ compared to their counterparts, $70.4 \%$ (Fig. 3).

The survival time of non-edematous children was longer than edematous children. At the end of the study, the hazard of death for those edematous children was $61.5 \%$ as compared to non-edematous children $16.9 \%$ ( Fig. 4).

\section{Predictors of mortality among severe acute malnourished children}

The relationship between the baseline variables and the risk of mortality was analyzed using bi-variable Cox proportional hazard regression model. From the bi-variable analysis, HIV/AIDS, pneumonia, TB, edema, Nasogastric tube feeding, chest in drawing, type of SAM, oxygen saturation below $90 \%$, altered pulse rate and respiratory rate ( at admission), failed appetite test, anemia (hgb $<11 \mathrm{mg} / \mathrm{dl}$ ), intake of F$100, W H Z, H A Z$, impaired consciousness at admission are predictors of mortality in SAM children. To identify independent predictors of mortality, multivariable Cox regression was performed for variables significant in bi-variable analysis. Only six variables oxygen saturation below $90 \%$, impaired consciousness at admission, intake of F-100, HIV/AIDS, edema and failed appetite test were significant predictors in the multivariable analysis.

The results of multivariable analysis showed that children who failed appetite test at admission were 2.44 hazard of death as compared to who have passed appetite test (AHR: $2.45 ; 95 \% \mathrm{Cl}: 1.28,4.69$ ). The risk of mortality of children who impaired consciousness level at admission were 2.25 times as compared to those conscious at admission (AHR:2.25; 95\%Cl: 1.08,4.68) (Table 4). 
Table 4

Results of bi-variable and multivariable Cox regression analysis of SAM children admitted at FHCSH from 2016-2019, Northwest Ethiopia, 2020.

\begin{tabular}{|c|c|c|c|c|c|}
\hline \multirow[t]{2}{*}{ Variables } & \multicolumn{3}{|c|}{ Survival Status } & \multirow[t]{2}{*}{$\mathrm{CHR}(95 \% \mathrm{Cl})$} & \multirow[t]{2}{*}{$\operatorname{AHR}(95 \% \mathrm{Cl})$} \\
\hline & & \multicolumn{2}{|c|}{$\begin{array}{l}\text { Event (\%) Censored } \\
\text { (\%) }\end{array}$} & & \\
\hline \multirow[t]{2}{*}{ HIV/AIDS } & Reactive & $13(41.9)$ & $18(58.1)$ & $2.83(1.49,5.36)$ & $\begin{array}{l}2.80(1.24 \\
6.34)^{\star}\end{array}$ \\
\hline & $\begin{array}{l}\text { Non- } \\
\text { Reactive }\end{array}$ & $37(10.7)$ & 308(89.3) & 1 & \\
\hline \multirow[t]{2}{*}{ TB } & Yes & 15(24.6) & $46(75.4)$ & $2.24(1.23,4.09)$ & $1.84(.88,3.85)$ \\
\hline & No & $39(9.5)$ & $371(90.5)$ & 1 & 1 \\
\hline \multirow[t]{2}{*}{ Pneumonia } & Yes & \multirow{2}{*}{$\begin{array}{l}33(17.2 \\
21(7.5)\end{array}$} & 159(82.8) & $2.45(1.41,4.24)$ & $0.95(0.45,2.00)$ \\
\hline & No & & $259(92.5)$ & 1 & 1 \\
\hline \multirow[t]{2}{*}{ oxygen saturation } & $<90 \%$ & $40(22.0)$ & 142(78.0) & $5.85(3.08,11.1)$ & $3.32(1.40,7 \cdot 88)^{\star}$ \\
\hline & $\geq 90 \%$ & $14(4.8)$ & 276(95.2) & 1 & 1 \\
\hline \multirow[t]{2}{*}{ Appetite test } & Failed & $31(25.0)$ & $93(75.0)$ & $4.2(2.45,7.33)$ & \multirow{2}{*}{$\begin{array}{l}2.45(1.28 \\
4.69)^{\star}\end{array}$} \\
\hline & Passed & $22(6.3)$ & $325(93.7)$ & 1 & \\
\hline \multirow{2}{*}{ WHZ } & Z-score<-3 & $40(13.9)$ & $248(86.1)$ & $2.09(1.13,3.85)$ & $1.52(0.69,3.41)$ \\
\hline & Z-score $\geq-3$ & $14(7.7)$ & 168(92.3) & 1 & 1 \\
\hline \multirow[t]{2}{*}{ IntakeofF-100 } & No & 23(19.5) & $95(80.5)$ & $2.96(1.71,5.10)$ & $2.63(1.35,5.12)^{\star}$ \\
\hline & Yes & $31(8.7)$ & $327(91.3)$ & 1 & 1 \\
\hline \multirow[t]{2}{*}{ NGT feeding } & Yes & $26(17.8)$ & $120(82.2)$ & $2.34(1.37,4.00)$ & $1.06(0.55,2.03)$ \\
\hline & No & $28(8.5)$ & $302(91.5)$ & 1 & 1 \\
\hline \multirow[t]{2}{*}{ Edema } & Yes & $36(19.2)$ & 151(80.8) & $3.05(1.73,5.37)$ & $2.85(1.44,5.64) *$ \\
\hline & No & $18(6.2)$ & 271(93.8) & 1 & 1 \\
\hline \multirow[t]{2}{*}{ Admission status } & Impaired & 16(51.6) & 15(48.4) & $6.66(3.69,12.0)$ & $2.25(1.08,4.68)^{\star}$ \\
\hline & Conscious & $38(8.54)$ & 407(91.46) & 1 & 1 \\
\hline \multirow{2}{*}{$\begin{array}{l}\text { Admission pulse } \\
\text { rate }\end{array}$} & Altered & $41(15.0)$ & 232(85.0) & $2.23(1.19,4.16)$ & $1.02(0.42,2.46)$ \\
\hline & Normal & $13(6.4)$ & 190(93.6) & 1 & 1 \\
\hline \multirow{2}{*}{$\begin{array}{l}\text { Admission } \\
\text { respiratory rate }\end{array}$} & Altered & $38(17.8)$ & 176(82.2) & $2.98(1.66,5.35)$ & $0.94(0.38,2.29)$ \\
\hline & Normal & $16(6.1)$ & 246(93.9) & 1 & 1 \\
\hline
\end{tabular}




\begin{tabular}{|llllll|}
\hline Variables & & Survival Status & CHR(95\% Cl) & AHR(95\%Cl) \\
\cline { 3 - 6 } & & \multicolumn{2}{c|}{$\begin{array}{l}\text { Event (\%) Censored } \\
(\%)\end{array}$} & & \\
\hline HAZ & Z-score<-3 & $33(14.7)$ & $192(85.3)$ & $2.01(1.16,3.48)$ & $1.60(0.75,3.42)$ \\
& Z-score $\geq-3$ & $21(8.5)$ & $225(91.5)$ & 1 & 1 \\
\hline Chest indrawing & Yes & $26(24.3)$ & $81(75.7)$ & $3.05(1.79,5.22)$ & $1.82(0.86,3.87)$ \\
& No & $28(7.7)$ & $337(92.3)$ & 1 & 1 \\
\hline Anemia & Yes & $33(15.5)$ & $180(84.5)$ & $1.67(.96,2.90)$ & $1.78(0.97,3.27)$ \\
& No & $21(8.0)$ & $242(92.0)$ & 1 & 1 \\
\hline
\end{tabular}

\section{Discussion}

This study revealed that the overall mortality of severe acute malnourished children was $11.34 \%$ with (955\% Cl: 8.40, 14.30) during the follow-up period. This finding is consistent with studies done, in Uganda (14), in Egypt (27) and studies conducted in Ethiopia, Jimma university (17), Gondar university (4) and Gedio Zone (28). On the other hand, this finding is lower than a study done in South Africa (12) and other study in Uganda (15). The discrepancy might be due to high proportion of HIV positive cases, in South Africa, $43 \%$ were HIV infected and this in turn increases the mortality due to the complexity of the management and opportunist infections. Similarly, the result is lower than the study conducted in Ethiopia, Sekota hospital (23), and Zewditu hospital (29).The difference can be due to differences in management team and supplies, the difference in the study period as there were changes in treatment modality and might be due to an increasing number of co-morbidity and severity of cases. However, the finding of this study was higher than a study in general hospital of Tigray (16). The possible justification of this discrepancy could be an increased complicated cases with co-morbidities like HIV/AIDS in our study which increased risk of death(12).

The overall incidence of mortality was found to be 9.1 death per 1000 person- days ( $95 \% \mathrm{Cl}$ : 6.97-11.88). This finding was in agreement with a study in Dilchora referral hospital 7.5 deaths/1000 person-days(25) and Gondar university 10.4 deaths /1000 person-days(20). On the contrary, the result was lower than a study done in Uganda 24 deaths/1000 person- days. This might be due to the difference in methodology which was a prospective cohort in the previous study. As a result, it included patients discharge following against medical advice and prior co-morbidities, for example in Uganda of the children were HIVinfected(15).

The survival probability was also found to be $90.9 \%$ and $72.8 \%$, at the 12 th and $20-25$ th days respectively. The overall cumulative survival probability at the end of 45 th days was $59.2 \%$. As result, median survival time could not determine. This study also indicated that the average length of stay in the hospital was 
12.5 days. This is in congruent with a study in Gondar University 12 days(4), Dilchora Hospital 10days(25), and international minimum (SPHERE) standard average length of wait that sets not exceed 30 days(30).

In the current study, SAM patients with oxygen saturation below $90 \%$ were more hazard of death as compared to those with saturation above $90 \%$. This result is supported by a finding in Uganda (14). This might be due to low oxygen saturation in the body causing respiratory failure, interference in brain and heart functions in SAM children(31).

SAM children presented with altered conscious level at admission were more hazard of death as compared to those conscious at admission. This was congruent with the finding in Jimma, Tigray and Kenya $(16,17,32)$. This might be because of a child with impaired conscious level increases the management complexity that is prone the children to nasogastric feeding, intravenous medication and fluid which were further reducing survival time $(2,5)$.

This study also revealed that not intake of F-100 was a significant predictor of mortality in SAM children. This is comparable with a study in Dilchora Hospital. The possible justification might be nutritional therapies (therapeutic feeding) are important in SAM children in reduction adaptation to maintain metabolic rate(5).

This study investigated that HIV infected children were 2.8 folds hazard of death as compared to HIVuninfected children. This was supported by a study conducted in Uganda Africa, Malawi and Zambia (11, $12,14,33)$. The result also in agreement with a study in Ethiopia(25). The possible reason could be HIV/AIDS together with malnutrition is particularly fatal because it increases management complexity and opportunistic infection which could impair feeding of SAM children(2, 3).

Failed appetite test at admission was 2.45 times hazard of death as compared to those passed appetite test and this result was comparable with similar studies $(14,25)$. The possible scientific justification might be, reduction in appetite is the sign of severe metabolic malnutrition and it is mainly metabolic malnutrition that causes death. A poor appetite means that the child has a significant infection or a major metabolic abnormality such as liver dysfunction, electrolyte imbalance and cell membrane damage. These are the patients at immediate risk of death(2).

In the current study Children presented with edema at admission were 2.9 times risk to die than nonedematous children. This finding is supported by a study done in Zambia(34). The possible justification could be due to edematous patients exhibit reduced ability to recover fluids, immune system failure, low lipid absorption and the liver is enlarged with fat so that, less ready to make glucose and exposed the child for hypoglycemia(1).

Although the current study did not show any significant association between severe acute malnutrition children with pneumonia, shock, TB, type of SAM, diarrhea, anemia, CHF and mortality, other investigations conducted in Zambia and South Africa $(11,12)$ and in Ethiopia $(17,23,25,28)$ showed 
that there was a significant association with child mortality. This discrepancy probably because of difference in sample size or might be the difference in the study period as there were changes in treatment modality and updating of professionals in standard training and regular supervision. The other possible justification could be strictly using the national management protocol, for example, TB and SAM management guidelines.

\section{Limitation of the study}

Since the data were retrospectively collected from patients' medical records, some important variables were excluded such as, parents' socio-demographic characteristics and the others may not recorded at all. Therefore, those threats might influence the outcome.

\section{Conclusion}

In this study, the overall survival status of severe acute malnourished children was low and incidence of mortality was high compared to international minimum (SPHERE) standard and previous reports in the literature. The major predictor of mortality were oxygen saturation below $90 \%$, not intake of F-100, HIV infection, edema, altered conscious level at admission and failed appetite test. Therefore, the health care workers should closely screen and give follow- up for severe acute malnourished children particularly those with identified predictors of death. Programmed monitoring and supervision concerning with national SAM standard management protocol is also highly recommended. Additionally longitudinal prospective follow up studies are recommended to identify additional socio-demographic factors, environmental factors and biochemical tests.

\section{Abbreviations}

AIDS: Acquired Immune Deficiency syndrome; AHR: Adjusted Hazard Ratio; Cl: Confidence Interval; HAZ: Height for Age Z-score; HIV: Human Immunodeficiency Virus; HR: Hazard Ratio; SAM: Severe Acute Malnutrition; WAZ: Weight for Age Z-score; WHZ: Weight for Height Z-score; IQR: Inter Quartile Range; WHO: World Health Organization; TFU: Therapeutic Feeding Unit.

\section{Declarations}

Ethical approval and consent to participate: Ethical clearance was obtained from Bahir Dar University, College of Medicine and Health Sciences, Ethical Clearance Review Committee namely (Taddesse Dagget, Yinager Workineh, Amlaku Mulat, Abirham Belachew and Chalachew Genet) with protocol number 0051/2020 and Institutional Review Board (IRB) decision number 002. Then, data were collected after getting consent from the hospital manager. This study didn't inflict or exposes children to unnecessary risk as a result of reviewing their medical records. Confidentiality of data was kept at all levels of the study and the data was not used for other purposes other than for this study.

\section{Consent of publication: Not applicable}


Availability of data and materials: Data will be available upon request from the corresponding author.

Competing interests: The authors declare that they have no competing interests.

Funding: This research didn't receive any grant from any funding agency in the public, commercial or notfor-profit sectors.

Authors' Contribution: AW, worked on the conception of the research idea, designing the study, involved in proposal writing, training and supervising the data collectors, analyzed and interpreting the results and preparing the manuscript. DA, MB, AT, SZ, GA and DK played their role in critically revising the proposal, participated in its design, analyzed and interpreting the results and wrote the manuscript. All authors were involved in reading and approving the final manuscript.

Acknowledgment: The authors acknowledged Bahir Dar University, College of Medicine and Health Sciences for support and facilitating of this study. The authors also acknowledged data collectors, supervisor and card room runners for their contribution of this work.

\section{References}

1. ROBERT M. KLIEGMAN MJWSGI M, SAMIR S. SHAH, MD, MSCE. Nelsontextbook of pediatrics. 21st ed. Canada.2018.

2. EMOH. Guidline for the managment of sever acute malnutrition. Addis Ababa, Ethiopia.2016.

3. UNICEF. The state of the worla's children ,2019. Children, food and nutrition. 2019.

4. Wagnew F, Tesgera D, Mekonnen M, Abajobir AA. Predictors of mortality among under-five children with severe acute malnutrition, Northwest Ethiopia: an institution based retrospective cohort study. 2018;76:64.

5. WHO.Guidline. Updates on the management of severe acute malnutrition in infants and children.World Health Organization. Geneva.2013.

6. UNICEF W, World Banck Group. Levels and Trend in child malnutrition. 2018.

7. You D, Jones G, Wardlaw T. United Nations Inter-agency Group for Child Mortality Estimation. Levels \& trends in child mortality: estimated developed by the UN Inter-agency Group for Child Mortality Estimation. New York: UNICEF. 2010. 2011.

8. Black RE, Victora CG, Walker SP, Bhutta ZA, Christian P, De Onis M, et al. Maternal and child undernutrition and overweight in low-income and middle-income countries. The lancet. 2013;382(9890):427-51.

9. Shanka N, Lemma S, Abyu D. Recovery rate and determinants in treatment of children with severe acute malnutrition using outpatient therapeutic feeding program in Kamba District, South West Ethiopia. 2015;5(2):155.

10. Desyibelew HD, Fekadu A, Woldie H. Recovery rate and associated factors of children age 6 to 59 months admitted with severe acute malnutrition at inpatient unit of Bahir Dar Felege Hiwot Referral 
hospital therapeutic feeding unite, northwest Ethiopia. PloS one. 2017;12(2):e0171020.

11. Munthali T, Jacobs $C$, Sitali $L$, Dambe R, Michelo $C$. Mortality and morbidity patterns in under-five children with severe acute malnutrition (SAM) in Zambia: a five-year retrospective review of hospitalbased records (2009-2013). Archives of Public Health. 2015;73(1):23.

12. Muzigaba M, Puoane T, Sartorius $B$, Sanders D. Independent and interactive effects of HIV infection, clinical stage and other comorbidities on survival of children treated for severe malnutrition in rural South Africa: A retrospective multicohort study. South African Journal of Child Health. 2017;11(1):4653.

13. Guesh G, Degu G, Abay M, Beyene B, Brhane E, Brhane K. Survival status and predictors of mortality among children with severe acute malnutrition admitted to general hospitals of Tigray, North Ethiopia: a retrospective cohort study. BMC Res Notes. 2018;11(1):832.

14. Nabukeera-Barungi N, Grenov B, Lanyero B, Namusoke H, Mupere E, Christensen VB, et al. Predictors of mortality among hospitalized children with severe acute malnutrition: a prospective study from Uganda. Pediatr Res. 2018;84(1):92-8.

15. Nalwanga D, Musiime V, Kizito S, Kiggundu JB, Batte A, Musoke P, et al. Mortality among children under five years admitted for routine care of severe acute malnutrition: a prospective cohort study from Kampala, Uganda. BMC pediatrics. 2020;20:1-11.

16. Guesh G, Degu G, Abay M, Beyene B, Brhane E, Brhane K. Survival status and predictors of mortality among children with severe acute malnutrition admitted to general hospitals of Tigray, North Ethiopia: a retrospective cohort study. 2018;11(1):832.

17. Jarso $\mathrm{H}$, Workicho A, Alemseged F. Survival status and predictors of mortality in severely malnourished children admitted to Jimma University Specialized Hospital from 2010 to 2012, Jimma, Ethiopia: a retrospective longitudinal study. BMC pediatrics. 2015;15:76.

18. EFMOH. National Strategy for Newborn and Child Survival in Ethiopia,2015/16-2019/2020. Addis Ababa.2015.

19. EPHI. Ethiopia Mini Demographic and Health Survey, 2019. Addis Ababa,Ethipia.2019.

20. Wagnew F, Tesgera D, Mekonnen M, Abajobir AA. Predictors of mortality among under-five children with severe acute malnutrition, Northwest Ethiopia: an institution based retrospective cohort study. Archives of public health $=$ Archives belges de sante publique. 2018;76:64.

21. Desyibelew HD, Baraki AG, Dadi AF. Mortality rate and predictors of time to death in children with severe acute malnutrition treated in Felege-Hiwot Referral Hospital Bahir Dar, Northwest Ethiopia. BMC research notes. 2019;12(1):409.

22. Hassen SL, Astatkie A, Mekonnen TC, Bogale GG. Survival Status and Its Determinants among UnderFive Children with Severe Acute Malnutrition Admitted to Inpatient Therapeutic Feeding Centers in South Wollo Zone, Amhara Region, Ethiopia. Journal of nutrition and metabolism. 2019;2019.

23. Desta K. Survival status and predictors of mortality among children aged 0-59 months with severe acute malnutrition admitted to stabilization center at Sekota Hospital Waghemra Zone. 2015;5:160. 
24. Chisti MJ, Salam MA, Ashraf H, Faruque AS, Bardhan PK, Hossain MI, et al. Clinical risk factors of death from pneumonia in children with severe acute malnutrition in an urban critical care ward of Bangladesh. PloS one. 2013;8(9):e73728.

25. Oumer A, Mesfin F, Demena M. Survival Status and Predictors of Mortality among Children Aged 0-59 Months Admitted with Severe Acute Malnutrition in Dilchora Referral Hospital, Eastern Ethiopia. East African Journal of Health and Biomedical Sciences. 2016;1(1):13-22.

26. Alebel A, Wagnew F, Tesema C, Kibret GD, Petrucka P, Eshite S. Effects of undernutrition on survival of human immunodeficiency virus positive children on antiretroviral therapy. Italian journal of pediatrics. 2018;44(1):29.

27. Ghazawy ER, Bebars GM, Eshak ES. Survival status and mortality predictors among severely malnourished under 5 years of age children admitted to Minia University maternity and children hospital. BMC pediatrics. 2020;20(1):1-10.

28. Girum T, Kote M, Tariku B, Bekele H. Survival status and predictors of mortality among severely acute malnourished children $<5$ years of age admitted to stabilization centers in Gedeo Zone: a retrospective cohort study. Therapeutics and clinical risk management. 2017;13:101.

29. Moges T, Haidar J. Management and outcome of severely malnourished children admitted to Zewditu Memorial Hospital, Ethiopia. East African journal of public health. 2009;6(2):162-7.

30. Layton R. The Sphere Project: Humanitarian charter and minimum standards in disaster response [book review]. Australian Journal of Emergency Management, The. 2001;16(1):37.

31. Sugiharto A, Firdausi R, Safitry 0 . The effects of declined oxygen levels on hypoxia symptoms and blood gases: An experimental study. 2018;1073(4):042023.

32. Maitland K, Berkley JA, Shebbe M, Peshu N, English M, Newton CR. Children with severe malnutrition: can those at highest risk of death be identified with the WHO protocol? PLoS medicine. 2006;3(12):500.

33. Chinkhumba J, Tomkins A, Banda T, Mkangama C, Fergusson P. The impact of HIV on mortality during in-patient rehabilitation of severely malnourished children in Malawi. Transactions of the Royal Society of Tropical Medicine and Hygiene. 2008;102(7):639-44.

34. Moramarco S, Amerio G, Kasengele Chipoma J, Nielsen-Saines K, Palombi L, Buonomo E. Filling the Gaps for Enhancing the Effectiveness of Community-Based Programs Combining Treatment and Prevention of Child Malnutrition: Results from the Rainbow Project 2015-17 in Zambia. International journal of environmental research and public health. 2018;15(9):1807.

\section{Figures}




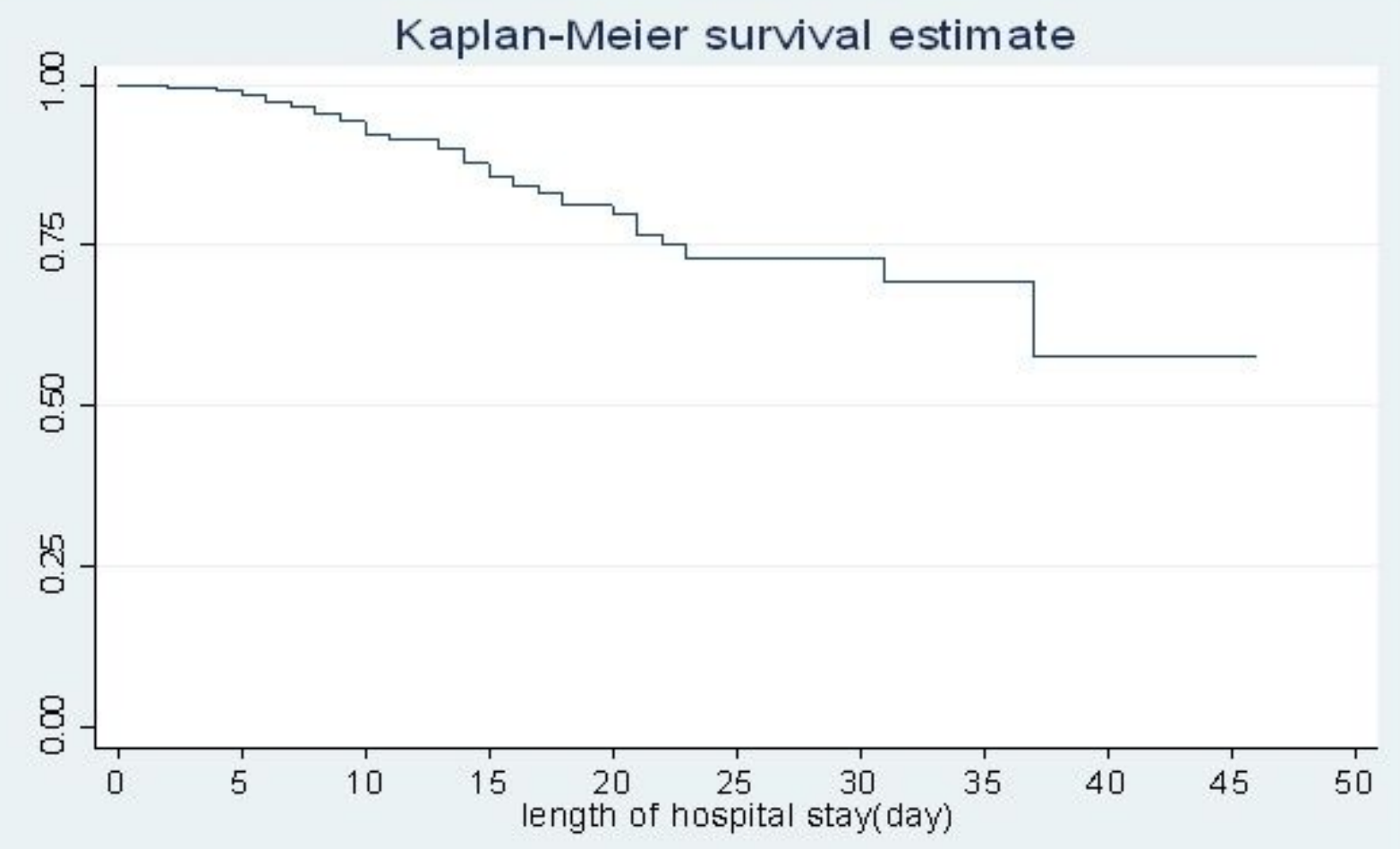

Figure 1

Overall Kaplan-Meier survival estimate of SAM children admitted to TFU of FHCSH, from 2016-2019, Northwest Ethiopia, 2020.

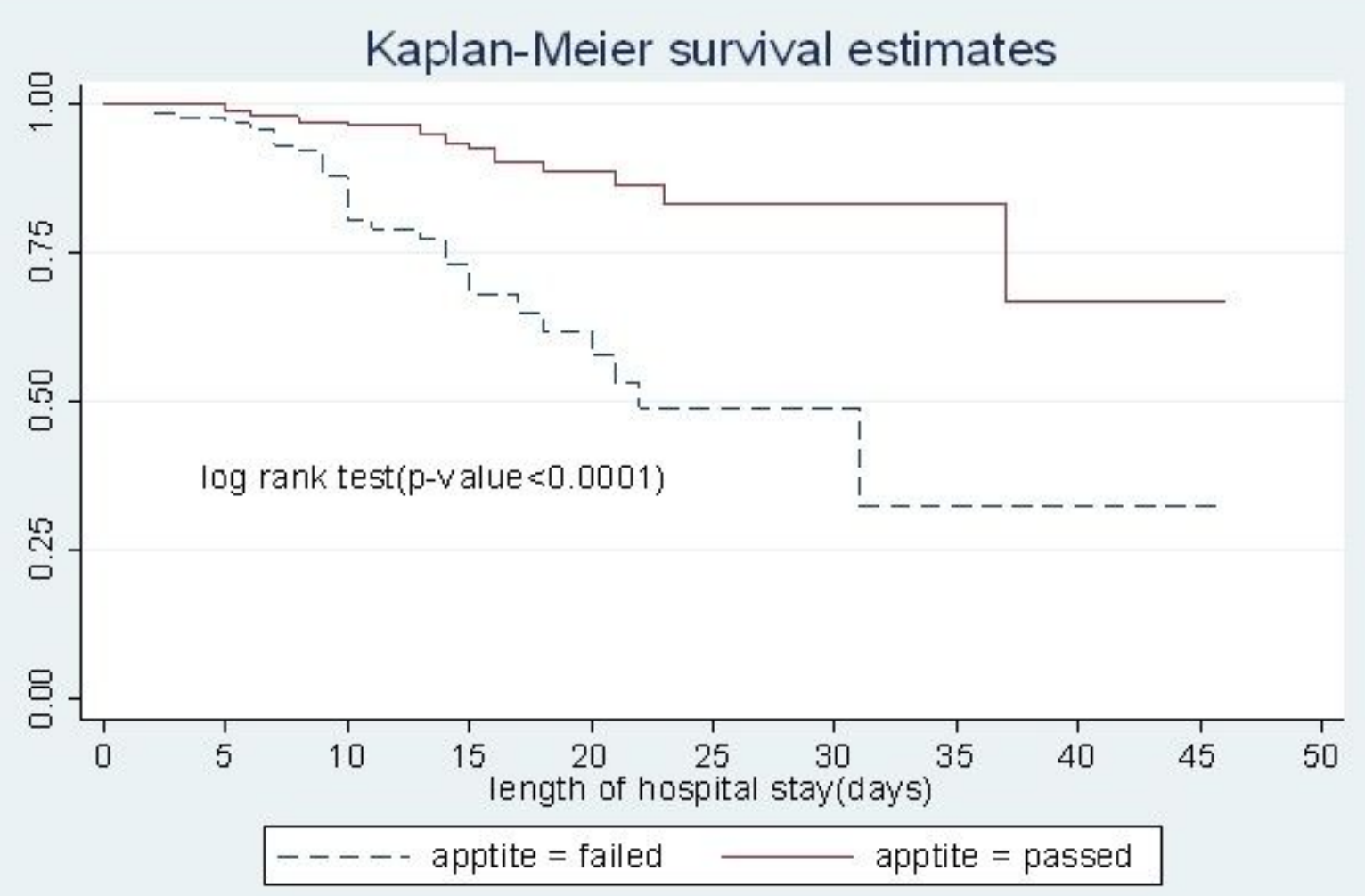


Figure 2

The Kaplan-Meier survival curves compare the survival time of SAM children admitted with categories of appetite test to TFU of FHCSH, from 2016-2019, North West Ethiopia, 2020.

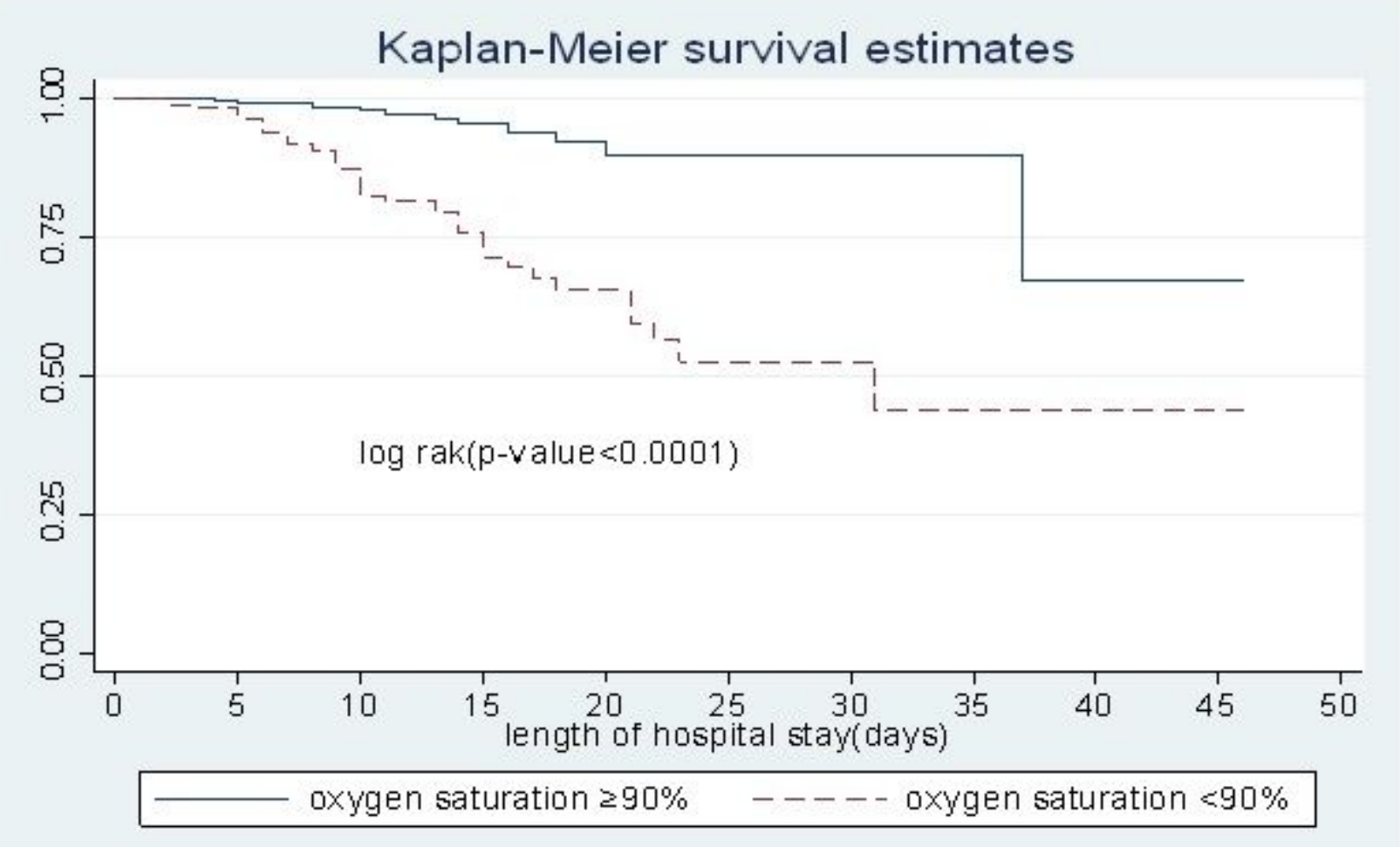

\section{Figure 3}

The Kaplan-Meier survival curves compare the survival time of SAM children admitted with categories of oxygen saturation level to TFU of FHCSH, from 2016-2019, Northwest Ethiopia, 2020. 


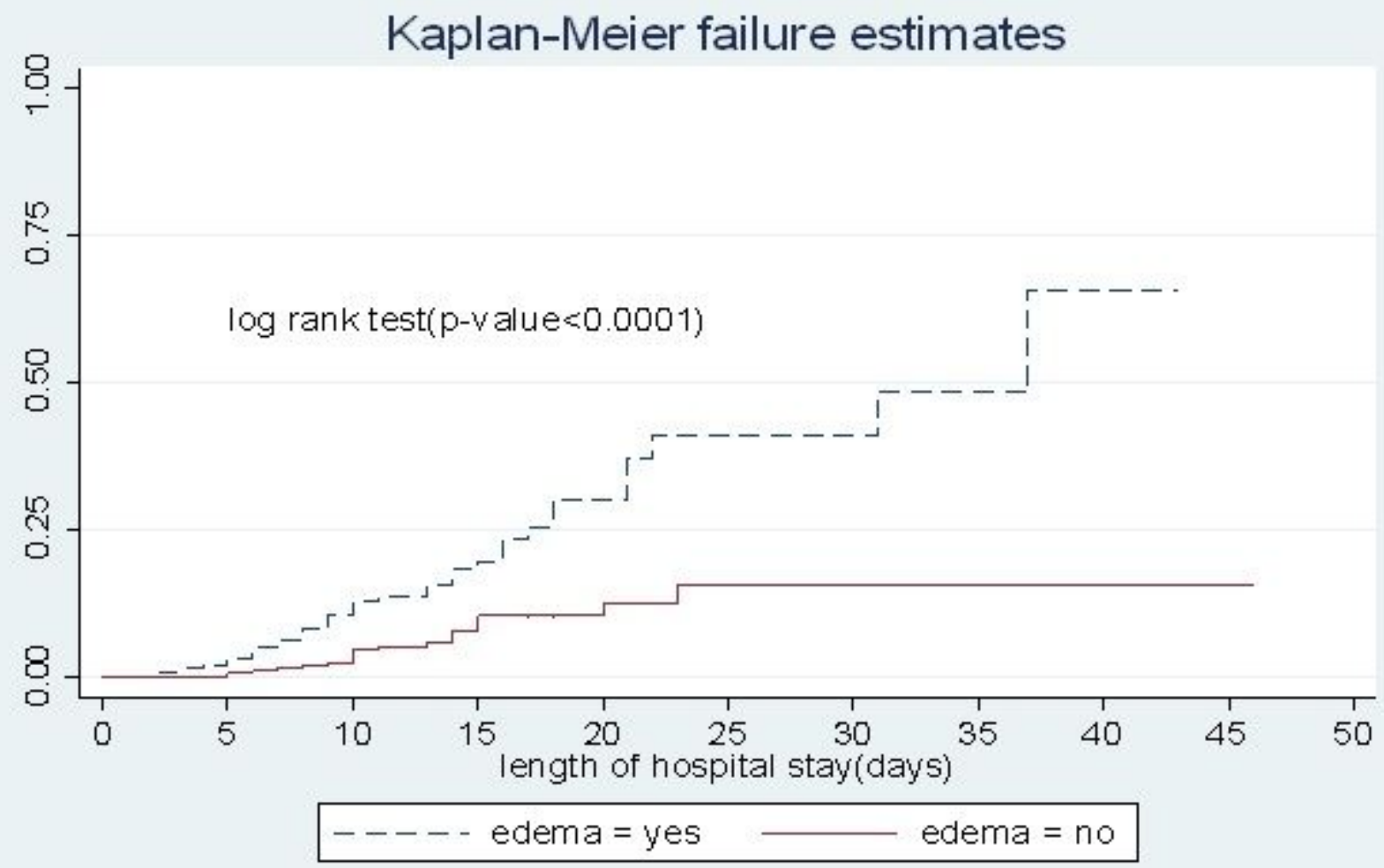

\section{Figure 4}

The Kaplan-Meier hazard curves compare hazard time of edematous children with Categories of nonedematous children, to TFU of FHCSH, from 2016-2019, Northwest Ethiopia, 2020 